\title{
The use of intralesional and posterior tibial nerve pulsed radiofrequency in the treatment of calcaneal spur
}

\author{
Ender Sır ${ }^{1}$, Sami Eksert ${ }^{2}$
}

(1) University of Health Sciences, Gulhane Training and Research Hospital, Algology and Pain Medicine Department, Ankara, Turkey

(2) University of Health Sciences, Gulhane Training and Research Hospital, Anesthesia and Reanimation Department, Ankara, Turkey

Date submitted:

Aug 08, 2019

Date accepted:

Sep 11, 2019

Online publication date:

December 15, 2019

\section{Corresponding Author:}

Sami Eksert

University of Health Sciences, Gulhane Training and Research Hospital, Algology and Pain Medicine Department, Ankara, Turkey exert79@yahoo.com

Keywords: Heel spur, posterior tibial nerve, pulsed radiofrequency, intractable pain, plantar fasciitis.

\begin{abstract}
Aims:Heel spur is a painful disease that adversely affects the quality of life of many people in different age groups. Many treatment methods have been described for pain management, and new techniques continue to be defined. In this study, we aimed to investigate the effectiveness of pulsed radiofrequency(PRF) application into the lesion and the posterior tibial nerve in the treatment of heel spur pain.
\end{abstract}

Methods:The medical records of 29 patients with chronic heel pain due to calcaneal spur and treated with PRF were reviewed. Group I received only intralesional PRF, Group II received both intralesional and posterior tibial nerve PRF. Numeric Rating Scale(NRS) was used to assess the pain level, The American Orthopedic Foot and Ankle Score(AOFAS) for the functional capacity, and the Likert score was used for the patient satisfaction. Measurements were performed before the procedure, at 3 weeks and 3 months after the procedure.

Results:Fifteen and fourteen patients were included in Group I and Group II, respectively. In both groups, NRS, AOFAS, and Likert scores at 3 weeks and 3 months were significantly improved when compared to pre-procedure values $(p<0.001)$. However, there was no statistically significant difference between the groups in terms of NRS, AOFAS and Likert scores $(p>0.05)$.

Conclusions:According to the results of the study, it was observed that the posterior tibial nerve PRF administration in addition to intralesional PRF, did not contribute to the reduction of pain and increase of functional capacity. Besides, it was concluded that intralesional PRF alone had good enough results in the management of heel spur pain.

\section{Introduction}

Heel pain is a clinical symptom of a variety of pathologies, including plantar fasciitis, calcaneal spur, or bursitis (1). Among these disorders, the calcaneal spur is the cause of approximately $15-20 \%$ of this intractable pain (2). Calcaneal spur, also known as heel spur or epin calcanei, is an anatomical malformation in the form of protrusion. Actually, the formation of the spur is secondary to the mechanical bone traction caused by plantar fasciitis (3), and mostly seen in patients who have high and long-standing activities such as runners, house workers, waiters, etc. (2-4). Also, obese people with a sedentary lifestyle have a higher incidence of degenerative foot pathologies, especially the calcaneal spur.

Several different treatment modalities are used in the treatment of heel spur pain, which is not rarely seen in the community. More invasive treatments can be preferred in patients unresponsive to conservative treatments such as pharmacological medications, physical therapy, and acupuncture. Radiotherapy, extracorporeal shock wave therapy, radiofrequency (RF) modalities and surgery are used in these patients who have re- fractory heel pain (5). Although there are many studies where $\mathrm{RF}$ ablation is used reliably, in recent years, there has been an increasing tendency to use PRF instead of conventional RF ablation to avoid complications associated with thermal lesion formation (6).

In clinical practice, patients have tenderness on their heels, and the pathology is often painful enough to make it difficult to perform daily activities and even extends to walking inability. The pain is felt on the inner and lower aspect of the heel and increases in proportion to the time the load is applied to the heel. The choice of treatment method is challenging for clinicians because of the complex anatomy of the region. The medial calcaneal nerve and the lateral plantar nerve are the terminal branches of the posterior tibial nerve, and they provide sensorial innervation of the heel of the foot. There are studies in which block or RF is applied to these nerves and the spur, as treatment methods with increasing interest and satisfactory results. (7-10). However, there is still controversy about where to administer PRF intervention, whether it should be applied to the lesion with surrounding tissue alone or combination with the sensorial nerve of the region. 
In this study, we aimed to compare the efficacy of intralesional PRF alone with the combination of intralesional PRF, and posterior tibial nerve PRF.

\section{Methods}

This retrospective study included 29 patients who have heel spurs and treated with PRF of the lesion and the posterior tibial nerve between January 2016 and December 2018. All patients underwent the procedure after obtaining written informed consent. Ethical approval was obtained from the Institutional Review Board (Health Sciences University, Gulhane Training and Research Hospital Ethics Committee. 2019/07, 19/130, Date: 09.04.2019).

Patients who had persistent heel pain due to calcaneal spur were divided into two groups. In Group I, percutaneous, fluoroscopy-guided intralesional PRF was applied to 15 patients. In Group II, 14 patients underwent percutaneous, fluoroscopy-guided posterior tibial nerve PRF, and intralesional PRF. Patients with heel spur pain for at least three months who did not respond to conservative treatments such as physiotherapy, nonsteroidal anti-inflammatory drugs and intralesional steroid injections were included in the study. Besides, patients with other causes of heel pain, including tarsal tunnel syndrome, Achilles tendinopathy, Haglund disease, calcaneal stress fracture were excluded.

The diagnosis was based on the clinical history and physical examination, which were supported with radiographic imaging of the calcaneal spur. The mode of treatment modalities was explained to all patients. Both groups were assessed using numerical verbal rating score (NRS) to evaluate the pain relief, ranging from none (0) to the extreme (10). The American Orthopedic Foot and Ankle Score (AOFAS), including a 9-item scale, was used to assess the improvement in foot functions (11). Patient satisfaction was evaluated with a 5-point Likert Scale, with 1 being the lowest and 5 being the highest level. The patients were evaluated with the above-mentioned scores before treatment and at 3 weeks and 3 months after treatment. None of the patients experienced adverse events or complications such as drowsiness, numbness, paresthesia, neuralgia, and motor weakness during the follow-up period.

\section{Technique}

Two investigators experienced in fluoroscopy-guided pain management interventions applied intralesional and dorsal tibial nerve PRF procedures under local anaesthesia. All patients were placed in the prone position, routine monitorization of pulse oximetry, electrocardiogram and noninvasive arterial pressure were performed, and intravenous access was established. After aseptic preparation using chlorhexidine, the calcaneal spur was visualized with fluoroscopy in each patient. Local anaesthesia of the skin was performed with a $25 \mathrm{G}$ (gauge) needle with $2 \mathrm{ml}, 2 \%$ lidocaine. A $5 \mathrm{~cm}, 22 \mathrm{G}, 10 \mathrm{~mm}$ active tip RF cannula (Model S-510, NeuroTherm, Inc., Middleton, MA, USA) introduced to the calcaneal spur (Figure 1A). Motor stimulation was performed at $1 \mathrm{~V}, 2 \mathrm{~Hz}$, with $1 \mathrm{~ms}$ pulse width, no motor response was observed in the foot muscles. When the sensory stimulation was applied with $50 \mathrm{~Hz}$ at a $1 \mathrm{~V}$ setting, the patient confirmed paresthesia in the heel region. Placement of the needle-tip was demonstrated via fluoroscopy (Figure 1B). After negative aspiration of blood, $1 \mathrm{ml}, 2 \%$ lidocaine was administered, followed by pulsed RF at $42{ }^{\circ} \mathrm{C}$ for 360 seconds.

In Group II, after aseptic preparation of both the heel and medial malleolus region of the foot, the intralesional PRF procedure in Group I was applied to all patients in Group II. Then, local anaesthesia of the skin to the posterior aspect of the medial malleolus of the foot was injected with a $25 \mathrm{G}$ needle. After then, a 22 G, $5 \mathrm{~cm}$ RF cannula (Model S-510, NeuroTherm, Inc., Middleton, MA, USA) with $10 \mathrm{~m}$ active tip was advanced to the posterior tibial nerve, and motor stimulation was applied at $2 \mathrm{~Hz}$ with a pulse width of $1 \mathrm{~ms}$ at $1 \mathrm{~V}$ (Figure $1 \mathrm{C})$. When the motor response was obtained by inversion, and plantar flexion of the foot, the stimulation reduced to 0.5 volts, and the appropriate motor response was ensured. Sensory stimulation was performed at $1 \mathrm{~V}$ with $50 \mathrm{~Hz}$, and the patient described paresthesia at the medial aspect of the heel and sole of the foot. After negative aspiration of blood, $1 \mathrm{ml}$ of $2 \%$ lidocaine was injected, then pulsed RF at $42^{\circ} \mathrm{C}$ performed for 360 seconds. After the procedure, patients were followed-up for 1 hour in terms of any complication.

\section{Statistical Analysis}

Statistical analysis was performed using Statistical Package for the Social Sciences (SPSS) version 21.0 (SPSS Inc., Chicago, IL, USA). Descriptive statistics were given as number, percentage, mean, standard deviation, minimum and maximum values. The continuous distribution of the data to the normal distribution was determined by the Kolmogorov Smirnov test.

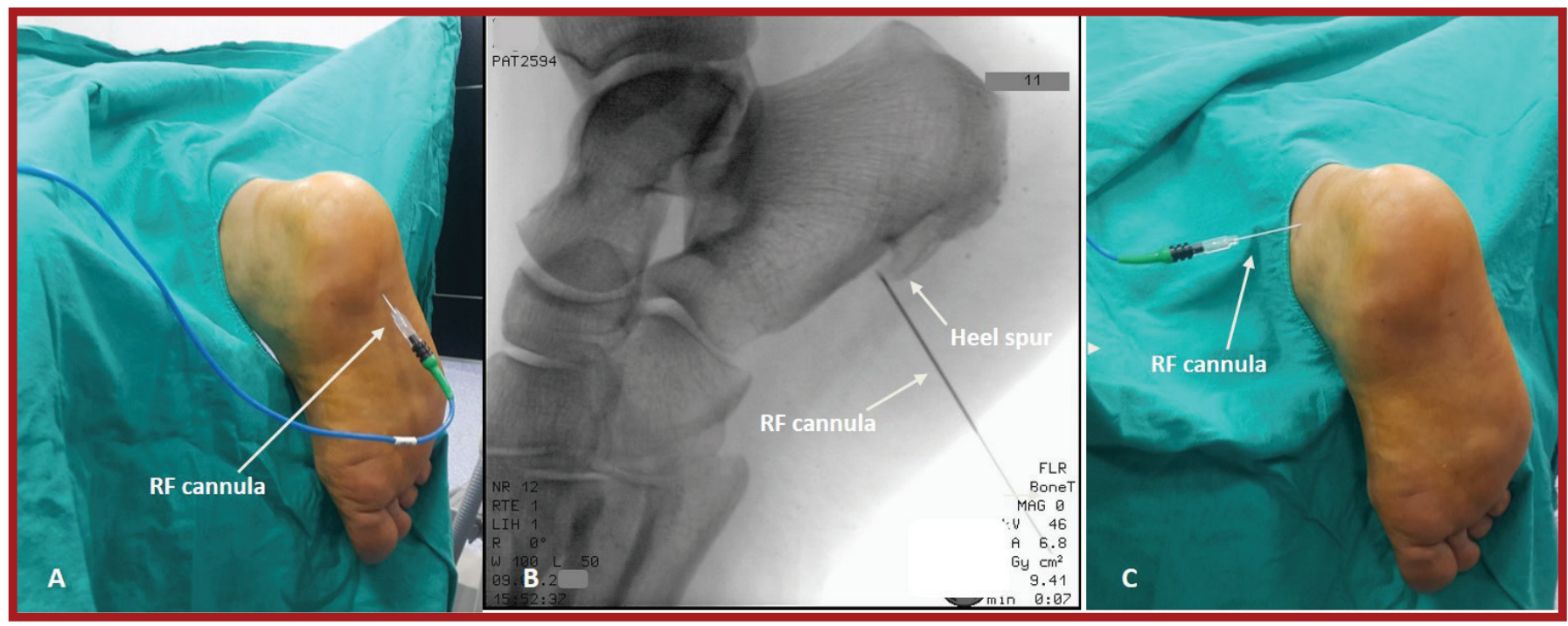


The Wilcoxon test was used to compare the continuous data in the dependent groups that did not conform to the normal distribution, and the Mann Whitney $U$ test was used to compare the independent groups. A p-value of $<0.05$ was considered statistically significant.

\section{Results}

Twenty-nine patients admitted to our clinic with the diagnosis of heel spurs and underwent intralesional PRF to the dorsal tibial nerve were included in the study. Fifteen patients in Group I received only intralesional PRF, while 14 patients in Group II received both intralesional and posterior tibial nerve PRF. In the study, the demographic characteristics of the patients were similar in both groups (Table 1). NRS scores were significantly improved at 3 weeks and 3 months when compared to pre-procedure values in both groups $(p<0.001)$, (Table 2$)$. When the AOFAS values were examined in both groups, a statistically significant increase was observed in the 3rd week and 3rd month scores compared to the pre-procedure $(p<0.001)$, (Table $3)$. Likewise, statistically significant improvement was observed in Likert scores in both groups at 3 weeks and 3 months after the procedure $(p<0.001)$, (Table 4$)$. However, when the NRS, AOFAS and Likert scores were examined, the differences between the groups were not statistically significant $(p>0.05)$.

Table 1. Demographic profile of the patients

\begin{tabular}{lccc}
\hline & Group I (n:15) & Group II (n:14) & p \\
\hline $\begin{array}{l}\text { Age (mean } \pm \text { SD), } \\
\text { year }\end{array}$ & $50.66 \pm 6.88$ & $52.64 \pm 8.12$ & 0.48 \\
\hline $\begin{array}{l}\text { Weight (mean } \pm \\
\text { SD), kg }\end{array}$ & $92.87 \pm 11.92$ & $82.07 \pm 11.17$ & 0.01 \\
\hline $\begin{array}{l}\text { Length (mean } \pm \\
\text { SD), cm }\end{array}$ & $163.73 \pm 8.45$ & $163.21 \pm 6.51$ & 0.18 \\
\hline BMI (mean \pm SD) & $34.68 \pm 3.91$ & $30.99 \pm 5.21$ & 0.04 \\
\hline
\end{tabular}

\section{Discussion}

In the current study, the efficacy of intralesional PRF alone was compared with the combination of intralesional PRF, and posterior tibial nerve PRF in terms of pain, functional capacity, and patient satisfaction. Both treatment protocols were found to be effective. However, when the three-month follow-up results were compared, no superiority of these two procedures to each other was observed in these parameters.

RF applications have been used for many years in the treatment of various pain syndromes (8). However, the use of RF in patients with chronic foot pain has recently been introduced (12). RF ablation acts by creating a thermal lesion, while PRF has a neuromodulatory effect on the target tissue and nerve (6). Because of the risk of conventional RF injury to the surrounding nerves and developing neuritis-like lesions, PRF has become more preferred by clinicians recently $(13,14)$. Although the mechanism of action of PRF is not fully elucidated, it has been suggested that the RF electrode generates an electromagnetic field in the tissue around the active tip (15). Also, changes in the expression of $\mathrm{C}$-fos gene by PRF is among the proposed mechanisms (16). Moreover, PRF is applied at low temperature and considered as a safe intervention with a lower risk of damaging healthy structures $(8,17)$. Thus, it can be proposed that the changes in the targeted nerve are not due to a heating effect but to an electro-mechanical and neuromodulatory effect. In the present study, PRF was applied instead of conventional RF ablation to avoid thermal injuries, and none of the patients showed any damage or complications in the nerve, and surrounding tissue and high success rates were achieved in both groups.

There are studies showing that not only to the nerve but also intra-articular, and intralesional PRF administration is beneficial in pain management $(7,18,19)$. In the current study, PRF treatment either on the lesion or the posterior tibial nerve innervating the heel region achieved effective analgesia after 3 weeks in all

Table 2. NRS scores of the patients before and after the treatment

\begin{tabular}{lccccc}
\hline & Group I (n:15) & Group II (n:14) & $\mathbf{p}^{*}$ & $\mathbf{p}^{* *}$ & $\mathbf{p}^{\text {***}}$ \\
\hline Pre-procedure NRS & $7.93 \pm 1.16$ & $7.71 \pm 1.06$ & - & - & 0.60 \\
\hline 3rd week NRS & $2.66 \pm 3.01$ & $3.00 \pm 2.25$ & $<0.001$ & $<0.001$ & 0.74 \\
\hline 3th month NRS & $2.93 \pm 3.08$ & $3.21 \pm 2.19$ & $<0.001$ & $<0.001$ & 0.77 \\
\hline $\begin{array}{l}\text { * Significance of the group I from pre-procedure value. } \\
\text { ** Significance of the group II from pre-procedure. } \\
\text { *** Significance between the group I and the group II. }\end{array}$ & & & & & \\
\hline
\end{tabular}

Table 3. AOFAS scores of the patients before and after the treatment

\begin{tabular}{lccccc}
\hline & Group I (n:15) & Group II (n:14) & $\mathbf{p}^{*}$ & $\mathbf{p}^{* *}$ & p \\
\hline Pre-procedure AOFAS & $40.20 \pm 10.61$ & $43.92 \pm 9.10$ & - & - & 0.32 \\
\hline 3rd week AOFAS & $79.20 \pm 18.59$ & $77.07 \pm 13.61$ & $<0.001$ & $<0.001$ & 0.72 \\
\hline 3th month AOFAS & $76.20 \pm 19.08$ & $76.35 \pm 13.60$ & $<0.001$ & $<0.001$ & 0.98 \\
\hline $\begin{array}{l}\text { * Significance of the group I from pre-procedure value. } \\
\text { ** Significance of the group II from pre-procedure. } \\
* * * \text { Significance between the group I and the group II. }\end{array}$ & & & & & \\
\hline
\end{tabular}

Table 4. Likert scores of the patients before and after the treatment

\begin{tabular}{lccccc}
\hline & Group I (n:15) & Group II (n:14) & $\mathbf{p}^{*}$ & $\mathbf{p}^{* *}$ & $\mathbf{p}^{* * *}$ \\
\hline Pre-procedure Likert & $1.46 \pm 0.51$ & $1.57 \pm 0.51$ & - & - & 0.58 \\
\hline 3rd week Likert & $4.00 \pm 1.30$ & $4.00 \pm 1.03$ & $<0.001$ & $<0.001$ & 0.98 \\
\hline 3th month Likert & $3.93 \pm 1.38$ & $3.85 \pm 0.94$ & $<0.001$ & $<0.001$ & 0.86 \\
\hline $\begin{array}{l}\text { * Significance of the group I from pre-procedure value. } \\
\text { ** Significance of the group II from pre-procedure. } \\
\star * \star \text { Significance between the group I and the group II. }\end{array}$ \\
\hline
\end{tabular}


patients and continued throughout the whole study period until 3 months. Furthermore, in a study by Liden et al., it was concluded that RF ablation of the sensory branches of the medial calcaneal nerve might be an alternative treatment for refractory heel pain. (20). Erken et al., and Landsman et al. supported this opinion by demonstrating the effectiveness of ablation of calcaneal branches of the inferior calcaneal nerve in their studies $(21,22)$. Also, successful results have been obtained in RF ablation of the lateral plantar nerve (9). The medial calcaneal nerve and the lateral plantar nerve are branches of the posterior tibial nerve and provide the sensorial innervation of the heel. Therefore, in addition to intralesional PRF, we applied PRF to the posterior tibial nerve in Group II patients. However, unlike RF procedures applied to the medial calcaneal and the lateral plantar branches, the application of PRF to the posterior tibial nerve did not contribute to the patients' outcomes in terms of pain and functionality.

Actually, the interventions seem similar in Group I, and Group II in this study. In methodology, we predicted that the combination of PRF of the lesion followed with the PRF of the posterior tibial nerve might be more effective. However, the results of pain relief in both showed no statistical difference. To the best of our knowledge, this is the first study comparing the effects of both techniques, which may change the perspectives of treatment strategies of the clinicians to the painful heel spur. Although there is no significant difference observed in the techniques we compare, there may be different combinations of treatment modalities to achieve more satisfactory outcomes in the future.

Nevertheless, there were some limitations to this study. First of all, we evaluated patients retrospectively, which may lead to the possibility of selection bias, and the lack of a control group in the study was limiting the strength of the current analysis. Second, the study population was small in both groups, and larger sample size could be better to find the frequency of complications associated with fluoroscopy-guided PRF applications. Third, the follow-up period of the patients in this study was 3 months, and this was a relatively short period, so, longer follow-up studies are needed to demonstrate the long-term results of the techniques applied in the study.

\section{Conclusions}

Our results show that fluoroscopy-guided intralesional PRF can yield promising results for painful heel spur. Furthermore, according to the preliminary results of our study, the administration of posterior tibial nerve PRF has no contribution to pain relief and functionality of patients who have calcaneal spurs, and only intralesional PRF is sufficient. However, in order to evaluate the efficacy and safety of these techniques in the treatment of heel spurs, we propose to conduct long-term, prospective clinical studies consisting of large series.

\section{Acknowledgments}

The authors would like to thank Dr Soykan Sahin for help with the statistical analysis of the manuscript.

\section{Conflict of Interest}

The authors declared that they do not have anything to disclose regarding conflict of interest with respect to this manuscript. There was no financial assistance with the project.

\section{References}

1. Agyekum EK, Ma K. Heel pain: A systematic review. Chi- nese Journal of Traumatology. 2015; 18(3):164-169.

2. Krukowska J, Wrona J, Sienkiewicz M, Czernicki J. A comparative analysis of analgesic efficacy of ultrasound and shock wave therapy in the treatment of patients with inflammation of the attachment of the plantar fascia in the course of calcaneal spurs. Arch Orthop Trauma Surg. Springer Berlin Heidelberg; 2016; 136(9):1289-1296.

3. Roxas M. Plantar fasciitis: diagnosis and therapeutic considerations. Altern Med Rev. 2005; 10(2):83-93.

4. Toomey EP. Plantar heel pain. Foot Ankle Clin. 2009; 14(2):229-245.

5. Uysal B, Beyzadeoglu M, Sager O, et al. Role of radiotherapy in the management of heel spur. Eur J Orthop Surg Traumatol. Springer Paris; 2015; 25(2):387-389.

6. Van Zundert J, de Louw AJA, Joosten EAJ, et al. Pulsed and continuous radiofrequency current adjacent to the cervical dorsal root ganglion of the rat induces late cellular activity in the dorsal horn. Anesthesiology. 2005; 102(1):125-131.

7. Ozan F, Koyuncu Ş, Gürbüz K, Öncel ES, Altay T. Radiofrequency Thermal Lesioning and Extracorporeal Shockwave Therapy: A Comparison of Two Methods in the Treatment of Plantar Fasciitis. Foot Ankle Spec. SAGE PublicationsSage CA: Los Angeles, CA; 2017; 10(3):204-209.

8. Osman AM, El-Hammady DH, Kotb MM. Pulsed Compared to Thermal Radiofrequency to the Medial Calcaneal Nerve for Management of Chronic Refractory Plantar Fasciitis: A Prospective Comparative Study. Pain Physician. 2016; 19(8):E1181-1187.

9. Arslan A, Koca TT, Utkan A, Sevimli R, Akel İ. Treatment of Chronic Plantar Heel Pain With Radiofrequency Neural Ablation of the First Branch of the Lateral Plantar Nerve and Medial Calcaneal Nerve Branches. J Foot Ankle Surg. 2016; 55(4):767-771.

10. Li X, Zhang L, Gu S, et al. Comparative effectiveness of extracorporeal shock wave, ultrasound, low-level laser therapy, noninvasive interactive neurostimulation, and pulsed radiofrequency treatment for treating plantar fasciitis: A systematic review and network meta-analysis. Medicine (Baltimore). 2018; 97(43):e12819.

11. Analay Akbaba Y, Celik D, Ogut RT. Translation, Cross-Cultural Adaptation, Reliability, and Validity of Turkish Version of the American Orthopaedic Foot and Ankle Society Ankle-Hindfoot Scale. J Foot Ankle Surg. 2016; 55(6):1139-1142.

12. Orhurhu V, Urits I, Orman S, Viswanath $O$, Abd-Elsayed A. A Systematic Review of Radiofrequency Treatment of the Ankle for the Management of Chronic Foot and Ankle Pain. Curr Pain Headache Rep. 2019; 23(1):4.

13. Erdem Y, Sir E. The Efficacy of Ultrasound-Guided Pulsed Radiofrequency of Genicular Nerves in the Treatment of Chronic Knee Pain Due to Severe Degenerative Disease or Previous Total Knee Arthroplasty. Med Sci Monit. International Scientific Information, Inc; 2019; 25:1857-1863.

14. Karaman H, Tüfek A, Kavak GO, Yildirim ZB, Celik F. 
Would pulsed radiofrequency applied to different anatomical regions have effective results for chronic pain treatment? J Pak Med Assoc. 2011; 61(9):879-885.

15. Brook J, Dauphinee DM, Korpinen J, Rawe IM. Pulsed radiofrequency electromagnetic field therapy: a potential novel treatment of plantar fasciitis. J Foot Ankle Surg. 2012; 51(3):312-316.

16. Higuchi Y, Nashold BS, Sluijter M, Cosman E, Pearlstein RD. Exposure of the dorsal root ganglion in rats to pulsed radiofrequency currents activates dorsal horn lamina I and II neurons. Neurosurgery. 2002; 50(4):8505-discussion856.

17. Thapa D, Ahuja V. Combination of diagnostic medial calcaneal nerve block followed by pulsed radiofrequency for plantar fascitis pain: A new modality. Indian J Anaesth. Medknow Publications; 2014; 58(2):183-185.

18. Eyigor C, Eyigor S, Akdeniz S, Uyar M. Effects of intra-articular application of pulsed radiofrequency on pain, functioning and quality of life in patients with advanced knee osteoarthritis. J Back Musculoskelet Rehabil. IOS Press; 2015; 28(1):129-134.

19. Gulec E, Ozbek H, Pektas S, Isik G. Bipolar Versus Unipolar Intraarticular Pulsed Radiofrequency Thermocoagulation in Chronic Knee Pain Treatment: A Prospective Randomized Trial. Pain Physician. 2017; 20(3):197-206.

20. Liden B, Simmons M, Landsman AS. A retrospective analysis of 22 patients treated with percutaneous radiofrequency nerve ablation for prolonged moderate to severe heel pain associated with plantar fasciitis. J Foot Ankle Surg. 2009; 48(6):642-647.

21. Erken HY, Ayanoglu S, Akmaz I, Erler K, Kiral A. Prospective study of percutaneous radiofrequency nerve ablation for chronic plantar fasciitis. Foot Ankle Int. 2014; 35(2):95-103.

22. Landsman AS, Catanese DJ, Wiener SN, Richie DH, Hanft JR. A prospective, randomized, double-blinded study with crossover to determine the efficacy of radio-frequency nerve ablation for the treatment of heel pain. J Am Podiatr Med Assoc. 2013; 103(1):8-15. 